\title{
Differential associations between diet and prediabetes or diabetes in the KORA FF4 study
}

\author{
Taylor A. Breuninger ${ }^{1,2,3,4} *$, Anna Riedl ${ }^{1,2,3,4}$, Nina Wawro ${ }^{1,2,3,4}$, Wolfgang Rathmann ${ }^{5}$, \\ Konstantin Strauch ${ }^{6,7}$, Anne Quante ${ }^{6,7}$, Annette Peters ${ }^{2,3}$, Barbara Thorand ${ }^{2,3}$, Christa Meisinger ${ }^{1,2,3,4}$ and \\ Jakob Linseisen ${ }^{1,2,3,4,8}$ \\ ${ }^{1}$ Helmboltz Zentrum München, German Research Center for Environmental Health (GmbH), Independent Research Unit Clinical Epidemiology, \\ Ingolstädter Landstr. 1, 85764 Neuherberg, Germany \\ ${ }^{2}$ Helmboltz Zentrum München, German Research Center for Environmental Health (GmbH), Institute of Epidemiology II, Ingolstädter Landstr. 1, \\ 85764 Neuherberg, Germany \\ ${ }^{3}$ German Center for Diabetes Research (DZD), München-Neuherberg, Ingolstädter Landstr. 1, 85764 Neuherberg, Germany \\ ${ }^{4}$ Ludwig-Maximilians-Universität München, Chair of Epidemiology, UNIKA-T Augsburg, Neusässer Str. 47, 86156 Augsburg, Germany \\ ${ }^{5}$ Deutsches Diabetes-Zentrum (DDZ), Institute for Biometrics and Epidemiology, Auf'm Hennekamp 65, 40225 Düsseldorf, Germany \\ ${ }^{6}$ Helmboltz. Zentrum München, German Research Center for Environmental Health (GmbH), Institute of Genetic Epidemiology, Ingolstädter \\ Landstr. 1, 85764 Neuberberg, Germany \\ ${ }^{7}$ Ludwig-Maximilians-Universität München, Chair of Genetic Epidemiology, IBE, Faculty of Medicine, 81377 Munich, Germany \\ ${ }^{8}$ ZIEL - Institute for Food and Health, Technical University of Munich, Weihenstephaner Berg 1, 85354 Freising, Germany
}

(Received 4 June 2018 - Final revision received 6 November 2018 - Accepted 9 November 2018)

Journal of Nutritional Science (2018), vol. 7, e34, page 1 of 12

doi:10.1017/jns.2018.25

Abstract

Type 2 diabetes mellitus (T2DM) is a global public health epidemic. Diet and lifestyle changes have been demonstrated as effective measures in managing T2DM and preventing or delaying the progression from prediabetes to diabetes, yet the relationship between diet, prediabetes and diabetes is still not entirely clear. The present study aimed to further elucidate the relationship between diet, diabetes and especially prediabetes. A total of 1542 participants of the cross-sectional, population-based Cooperative Health Research in the Region of Augsburg (KORA) FF4 study (2013/2014) were included in this analysis. Dietary intake was derived using a method combining information from a FFQ and repeated 24-h food lists. Glucose tolerance status was assessed via oral glucose tolerance tests in all participants without a previous physician-confirmed diagnosis of T2DM, and was classified according to the 2003 American Diabetes Association criteria. Crude and fully adjusted multinomial logistic regression models were fitted to examine associations between diet and prediabetes, undetected diabetes mellitus (UDM) and prevalent T2DM. After adjusting for major covariates, fruit was significantly inversely and total meat, processed meat, sugar-sweetened beverages and moderate alcohol significantly associated with UDM and/or prevalent diabetes. Sex-specific analyses showed that in men, coffee was significantly inversely (OR 0.80; $95 \%$ CI $0 \cdot 67,0 \cdot 96$ ) and heavy alcohol significantly (OR 1.84; $95 \%$ CI $1.14,2 \cdot 95)$ associated with prediabetes. Our findings on diet and T2DM are consistent with current literature, while our results regarding coffee, heavy alcohol consumption and prediabetes highlight new possible targets for primary prevention of the derangement of glucose homeostasis.

Key words: Alcohol: Coffee: Fruit: Meat: enable-Cluster

* Corresponding author: Taylor A. Breuninger, fax +49 893187 2951, email taylor.breuninger@helmholtz-muenchen.de

Abbreviations: 24HFL, 24-h food list; BVSII, Bavarian Food Consumption Survey II; KORA, Cooperative Health Research in the Region of Augsburg; NGT, normal glucose tolerance; SSB, sugar-sweetened beverages; T2DM, type 2 diabetes mellitus; UDM, undetected diabetes mellitus.

(C) The Author(s) 2018. This is an Open Access article, distributed under the terms of the Creative Commons Attribution licence (http://creativecommons.org/licenses/by/4.0/), which permits unrestricted re-use, distribution, and reproduction in any medium, provided the original work is properly cited. 
Type 2 diabetes mellitus (T2DM) is a major public health concern that places a significant burden on both the healthcare system and the individual. Costs associated with T2DM were estimated to be $€ 33.3$ billion in Germany in 2010, with total healthcare costs for people with diabetes estimated to be three times higher than for people without diabetes ${ }^{(1)}$. This disease is associated with numerous adverse outcomes and complications for the patient, especially concerning macro- and microvascular disease progression ${ }^{(2,3)}$. Prediabetes, the presence of blood glucose levels that are elevated above normal but are still below the threshold for T2DM, has been recognised as placing an individual at increased risk of developing T2 $\mathrm{DM}^{(4)}$. What is more, some evidence suggests that the state of prediabetes itself is not benign and may already be associated with complications such as increased CVD risk, retinopathy, neuropathy and nephropathy ${ }^{(5)}$. It is estimated that between 18 and $24 \%$ of the German population has prediabetes, and that $5-10 \%$ of people with prediabetes may progress to diabetes each year without intervention ${ }^{(6,7)}$.

Lifestyle alterations, including dietary changes, offer an estimated $40-70 \%$ reduction in the relative risk of the progression from prediabetes to diabetes ${ }^{(6)}$. Therefore, it is imperative to have a complete understanding of the impact that diet has on the risk of both prediabetes and diabetes in order to make effective dietary recommendations. While extensive, though not conclusive, research has been done on diet and the risk of diabetes, relatively little research has investigated the relationship between dietary intake and the risk of prediabetes $^{(8-12)}$. Therefore, the aim of the present study was to examine the cross-sectional association between dietary intake, prediabetes and T2DM in the Cooperative Health Research in the Region of Augsburg (KORA) FF4 study population, in order to better inform future prospective and experimental investigations.

\section{Methods}

\section{Study population}

The KORA FF4 study (2013/2014) is the second follow-up examination of the population-based KORA S4 health survey, conducted in the city of Augsburg and two surrounding counties in Southern Germany between 1999 and 2001. Of the 4261 individuals included in the initial survey, 2279 individuals also participated in the KORA FF4 study. Details regarding the participation rate for KORA FF4 have been published previously $^{(13)}$. Of the total 2279 individuals who participated in FF4, those with type 1 diabetes mellitus ( $n 6)$, unknown glucose tolerance status $(n 93)$ or missing dietary intake data ( $n$ 638) were excluded from this analysis. There were complete covariate data for all remaining 1542 participants. A sensitivity analysis comparing FF4 participants who were included $v$. excluded from the present analysis showed few differences, though participants who were excluded had a higher prevalence of smoking and were less active (data not shown). This study was conducted according to the guidelines laid down in the Declaration of Helsinki and all procedures involving human subjects were approved by the ethics committee of the Bavarian Chamber of Physicians in Munich. Written informed consent was obtained from all subjects.

\section{Assessment of glucose tolerance status}

Prevalent diabetes was defined by either current use of a glucose-lowering medication or a self-reported diagnosis of T2DM, both confirmed by the individual's physician. The remaining participants without a known diabetes diagnosis underwent a standard oral glucose tolerance test (OGTT) to classify them by glucose tolerance status according to the 2003 American Diabetes Association diagnostic criteria ${ }^{(14)}$. Further details have been described elsewhere ${ }^{(13)}$. Individuals with an OGT'T value of $\geq 7.0 \mathrm{mmol} / 1$ fasting or $\geq 11.1$ $\mathrm{mmol} / \mathrm{l} 2$-h glucose were classified with undetected diabetes mellitus (UDM), also known as screen-detected diabetes. Prediabetes was defined as having impaired fasting glucose (5.6-6.9 mmol/1 fasting glucose), impaired glucose tolerance $(7 \cdot 8-11.0 \mathrm{mmol} / 1 \mathrm{2}$-h glucose) or the combination of both. Normal glucose tolerance (NGT) was considered a fasting glucose value of $<5.6 \mathrm{mmol} / 1$ and a 2 -h glucose OGTT value of $<7.8 \mathrm{mmol} / \mathrm{l}$.

\section{Dietary assessment}

Dietary intake was assessed in KORA FF4 participants via repeated 24-h food lists (24HFL) and one FFQ. The 24HFL is a closed list of $>300$ food items used to evaluate which foods were consumed over the previous $24 \mathrm{~h}$. A detailed description has been provided elsewhere ${ }^{(15)}$. The FFQ was based on the German multilingual European Food Propensity Questionnaire (EFPQ) and encompassed approximately 148 food items and determined which foods were consumed, how frequently and in what amount over the past 12 months ${ }^{(16)}$. The first $24 \mathrm{HFL}$ was administered at the test centre. Participants were encouraged to complete web-based questionnaires to prevent the submission of incomplete data. However, a paper questionnaire could be made available upon request. A total of 1602 participants completed one FFQ and at least one 24HFL. Of these participants, 652 $(40.7 \%)$ completed two 24HFL and 826 (51.6\%) completed three $24 \mathrm{HFL}$.

Habitual dietary intake was then calculated for these 1602 participants using an approach based on the National Cancer Institute method, a validated method that has the goal of reducing the error associated with traditional dietary assessment tools. The National Cancer Institute method approximates habitual dietary intake based on both the probability of consuming a food on a given day and the usual portion size in which a food is consumed ${ }^{(17,18)}$. Following the idea of this twostep method, consumption probability was first estimated for each individual and each food item based on 24HFL data using logistic mixed models, which were adjusted for age, sex, BMI, physical activity, smoking, education and food consumption frequency, as evaluated by the FFQ. Because the 24HFL do not include consumption amounts, they were predicted based on data from 24-h diet recalls obtained from participants of the Bavarian Food Consumption Survey II 
(BVSII). The BVSII study was designed as a representative, population-based, cross-sectional study to characterise the dietary and lifestyle habits of Bavarians. Linear mixed models were used to calculate the consumption amount for a consumption day for each food item in the BVSII study. The models were adjusted for age, sex, BMI, physical activity, education and smoking. The $\beta$-estimates calculated for each food intake variable from the BVSII data were then used to predict the habitual consumption amount for each food item for each individual in the KORA FF4 study, based on his or her characteristics. Finally, the habitual dietary intake was calculated for each individual by multiplying the estimated intake probability for each food item by the estimated consumption amount. The dietary intake data were then categorised into sixteen food groups and twenty-one food subgroups according to the European Prospective Investigation into Cancer and Nutrition (EPIC)Soft classification scheme ${ }^{(19)}$. Nutrient intakes were derived from habitual food intake estimates using the National Nutrient Database (Bundeslebensmittelschlüssel; BLS 3.02). Fruits $(\mathrm{g} / \mathrm{d})$, vegetables $(\mathrm{g} / \mathrm{d})$, potatoes $(\mathrm{g} / \mathrm{d})$, total meat $(\mathrm{g} / \mathrm{d})$, red meat (beef and pork, $\mathrm{g} / \mathrm{d}$ ), poultry $(\mathrm{g} / \mathrm{d})$, processed meat $(\mathrm{g} / \mathrm{d})$, eggs $(\mathrm{g} / \mathrm{d})$, total dairy products $(\mathrm{g} / \mathrm{d})$, milk $(\mathrm{g} / \mathrm{d})$, yogurt $(\mathrm{g} / \mathrm{d})$, cheese $(\mathrm{g} / \mathrm{d})$, coffee $(\mathrm{g} / \mathrm{d})$, fruit and vegetable juice $(\mathrm{g} / \mathrm{d})$ and sugar-sweetened beverages $(\mathrm{SSB}, \mathrm{g} / \mathrm{d})$ are the food groups and subgroups which were used as the main exposure variables. Alcohol $(\mathrm{g} / \mathrm{d})$, total fibre $(\mathrm{g} / \mathrm{d})$ and insoluble fibre $(\mathrm{g} / \mathrm{d})$ are the nutrients that were chosen. Insoluble fibre was selected as an alternative to whole grain intake, which is not included in the EPIC-Soft classification system. These selections were made based on the findings of previous research that indicated either a positive or negative association with the consumption of these food items and the prevalence of $\mathrm{T} 2 \mathrm{DM}^{(8,20-29)}$. All selected food intake variables, excluding alcohol, were then divided by their standard deviation in order to give risk estimates per $1 \mathrm{sD}$. In accordance with the nutrient reference values of the German Nutrition Society (Deutsche Gesellschaft für Ernährung), alcohol intake was categorised as non-consumer ( $<5 \mathrm{~g} / \mathrm{d}$ for men, $<2 \mathrm{~g} / \mathrm{d}$ for women), moderate $(\geq 5-<20 \mathrm{~g} / \mathrm{d}$ for men and $\geq 2-<10 \mathrm{~g} / \mathrm{d}$ for women) or heavy consumption ( $\geq 20 \mathrm{~g} / \mathrm{d}$ for men and $\geq 10 \mathrm{~g} / \mathrm{d}$ for women), in order to examine potential differences in association between moderate and heavy alcohol intake ${ }^{(30)}$. Non-consumers were classified as $<2 \mathrm{~g} / \mathrm{d}$ or $<5 \mathrm{~g} / \mathrm{d}$ rather than $0 \mathrm{~g} / \mathrm{d}$ due to the method used for dietary intake estimation in this study, which does not allow for an intake of $0 \mathrm{~g} / \mathrm{d}$.

\section{Assessment of covariates}

Potential covariates were selected from the existing literature on diet and diabetes ${ }^{(20,24,31-33)}$. The selected covariates were age (years), sex (male/female), BMI $\left(\mathrm{kg} / \mathrm{m}^{2}\right)$, waist circumference $(\mathrm{cm})$, family history of diabetes (yes/no/do not know), physical activity (active $\geq 1 \mathrm{~h}$ per week/inactive), smoking (current/ex-/never smoker), hypertension $(\geq 140 / 90 \mathrm{mmHg}$ or antihypertensive medication, yes/no) and education. In accordance with the German education system, education was categorised as $<10$ years, $10-<13$ years and $\geq 13$ years for the analyses including all participants, but had to be condensed to $<13$ years and $\geq 13$ years for the analyses stratified by sex due to low frequencies in the $<10$ years group. Vocational training is also taken into account in this variable. Waist circumference, blood pressure and BMI were measured at the study centre in standardised fashion by trained examiners. Education (years), family history of diabetes, physical activity and smoking status (regular/occasional/ex-/never smoker) were assessed during a face-to-face interview conducted by trained investigators. Further details have been outlined elsewhere ${ }^{(13,34)}$.

\section{Statistical analysis}

Baseline characteristics were examined according to glucose tolerance status and sex. Characteristics were expressed as median and 25th and 75th percentiles or percentage and number. Significant differences between glucose tolerance status groups were evaluated using the Kruskal-Wallis test for continuous variables and the $\chi^{2}$ test for categorical variables. Fisher's exact test was used if frequencies were too low. Habitual dietary intake was also described according to glucose tolerance status and sex using the mean and standard deviation and median and 25th and 75th percentiles. Significant differences between glucose tolerance status groups were assessed using the Kruskal-Wallis test. To assess the associations between dietary intake and diabetes (NGT, prediabetes, UDM and prevalent T2DM), multinomial logistic regression was performed using the mlogit() function from the $\mathrm{R}$ package mlogit. Two multinomial logistic regression models were fitted for each dietary intake variable of interest with different sets of covariates: the crude model was adjusted for age, sex and energy intake; the fully adjusted model was additionally adjusted for BMI, waist circumference, family history of diabetes, physical activity, smoking, education and hypertension. All analyses were performed for the total sample of participants and stratified by sex. $P$ values $<0.05$ were considered statistically significant. All analyses were performed using RStudio version 1.0.143 and $\mathrm{R}$ version 3.4.0.

\section{Results}

Of the 1542 participants included in the analyses, $789(51.2 \%)$ had NGT, $545(35.3 \%)$ had prediabetes, sixty-five (4.2\%) had UDM and $143(9.3 \%)$ had prevalent diabetes. Table 1 displays the characteristics of these individuals according to sex and glucose tolerance status. There was a statistically significant difference between participants based on glucose tolerance status for all characteristics in both sexes. The participants with prediabetes, UDM and prevalent diabetes were older, less educated, had a higher BMI and waist circumference and were less active than individuals with NGT. Hypertension and a family history of diabetes were more prevalent in participants with prediabetes, UDM and prevalent diabetes than in individuals with NGT.

Table 2 characterises the dietary intake of men and women in this study according to glucose tolerance status, as well as the mean and standard deviation for each food item for the total population. Potato, processed meat, dairy products, 
Table 1. Characteristics of the study population* by glucose tolerance status and sex (Medians and 25th and 75th percentiles for continuous variables; percentages and numbers for categorical variables)

\begin{tabular}{|c|c|c|c|c|c|c|c|c|c|}
\hline & \multicolumn{2}{|c|}{ NGT } & \multicolumn{2}{|c|}{ Prediabetes } & \multicolumn{2}{|c|}{ UDM } & \multicolumn{2}{|c|}{ Prevalent diabetes } & \multirow[b]{2}{*}{$P \dagger$} \\
\hline & Median/\% & $25 \%, 75 \% / n$ & Median/\% & $25 \%, 75 \% / n$ & Median/\% & $25 \%, 75 \% / n$ & Median/\% & $25 \%, 75 \% / n$ & \\
\hline \multicolumn{10}{|l|}{$\operatorname{Sex}(n)$} \\
\hline Men & 292 & & 333 & & 36 & & 91 & & $<0.001$ \\
\hline Women & 497 & & 212 & & 29 & & 52 & & \\
\hline \multicolumn{10}{|c|}{ Age (years) } \\
\hline Men & 54 & 47,64 & 62 & 53,71 & 70 & 65,76 & 72 & 66,76 & $<0.001$ \\
\hline Women & 53 & 46,62 & 63 & 57,71 & 67 & 65,77 & 68 & 63,76 & $<0.001$ \\
\hline \multicolumn{10}{|c|}{ Education (<13 years) } \\
\hline Men & 51.4 & 150 & $60 \cdot 1$ & 200 & $75 \cdot 0$ & 27 & 74.7 & 68 & $<0.001$ \\
\hline Women & $61 \cdot 0$ & 303 & $76 \cdot 9$ & 163 & 75.9 & 22 & $78 \cdot 8$ & 41 & $<0.001$ \\
\hline \multicolumn{10}{|c|}{ BMI $\left(\mathrm{kg} / \mathrm{m}^{2}\right)$} \\
\hline Men & $26 \cdot 2$ & $24 \cdot 3,28 \cdot 6$ & $28 \cdot 1$ & $25 \cdot 7,30 \cdot 9$ & $29 \cdot 3$ & $27 \cdot 0,32 \cdot 4$ & 29.6 & $26 \cdot 5,33 \cdot 4$ & $<0.001$ \\
\hline Women & $24 \cdot 7$ & $22 \cdot 3,27 \cdot 6$ & 28.5 & $25 \cdot 3,31 \cdot 6$ & 31.5 & $26 \cdot 4,34.5$ & 31.4 & $28 \cdot 6,35 \cdot 8$ & $<0.001$ \\
\hline \multicolumn{10}{|c|}{ Waist circumference $(\mathrm{cm})$} \\
\hline Men & 97 & 91,104 & 103 & 97,111 & 107 & 101,113 & 108 & 101,118 & $<0.001$ \\
\hline Women & 84 & 77,94 & 95 & 87,104 & 99 & 93,110 & 105 & 95,112 & $<0.001$ \\
\hline \multicolumn{10}{|c|}{ Physical activity (active) } \\
\hline Men & $65 \cdot 1$ & 190 & $59 \cdot 2$ & 197 & $30 \cdot 6$ & 11 & $36 \cdot 3$ & 33 & $<0.001$ \\
\hline Women & $69 \cdot 8$ & 347 & $61 \cdot 3$ & 130 & $48 \cdot 3$ & 14 & $48 \cdot 1$ & 25 & $<0.001$ \\
\hline \multicolumn{10}{|c|}{ Smoking (current smoker)‡ } \\
\hline Men & $16 \cdot 8$ & 49 & 13.5 & 45 & 8.3 & 3 & 8.8 & 8 & 0.004 \\
\hline Women & $15 \cdot 1$ & 75 & $13 \cdot 2$ & 28 & 0.0 & 0 & $5 \cdot 8$ & 3 & 0.005 \\
\hline \multicolumn{10}{|c|}{ Smoking (ex-smoker)‡ } \\
\hline Men & $44 \cdot 2$ & 129 & $55 \cdot 0$ & 183 & 55.6 & 20 & $68 \cdot 1$ & 62 & \\
\hline Women & 39.4 & 196 & 33.0 & 70 & $27 \cdot 6$ & 8 & $28 \cdot 8$ & 15 & \\
\hline \multicolumn{10}{|c|}{ Family history of diabetes (yes)‡ } \\
\hline Men & $23 \cdot 3$ & 68 & 24.9 & 83 & $27 \cdot 8$ & 10 & 39.6 & 36 & 0.007 \\
\hline Women & $24 \cdot 1$ & 120 & 35.4 & 75 & $31 \cdot 0$ & 9 & $36 \cdot 5$ & 19 & $<0.001$ \\
\hline \multicolumn{10}{|c|}{ Hypertension (yes) } \\
\hline Men & $27 \cdot 8$ & 81 & 49.5 & 165 & $83 \cdot 3$ & 30 & 74.7 & 68 & $<0.001$ \\
\hline Women & $20 \cdot 8$ & 103 & $46 \cdot 2$ & 98 & $55 \cdot 2$ & 16 & $75 \cdot 0$ & 39 & $<0.001$ \\
\hline
\end{tabular}

NGT, normal glucose tolerance; UDM, undetected diabetes mellitus.

${ }^{*} n 1542$ (men, $n$ 752; women, $n$ 790).

$\dagger P$ values were calculated using the Kruskal-Wallis test for continuous variables and the $\chi^{2}$ test for categorical variables.

¥ Fisher's exact test was used to calculate $P$ values due to low frequencies.

milk, energy and alcohol intake differed significantly according to glucose tolerance status in both men and women. Egg, coffee and juice consumption varied significantly according to glucose tolerance status in men; and vegetable, yogurt, total meat and red meat consumption differed significantly between groups in women.

Table 3 shows the results of the crude multinomial logistic regression models. In the total study sample, fruit, vegetables, total meat, red meat, processed meat, total dairy products, yogurt, coffee, SSB, moderate alcohol, total fibre and insoluble fibre had statistically significant associations with glucose tolerance status. When stratified by sex, there was a significant association for total meat, red meat, processed meat, SSB, total fibre and insoluble fibre for both men and women. Coffee and heavy alcohol remained significant only for men, while yogurt and moderate alcohol remained significant only for women. The results of the fully adjusted models are presented in Table 4. A significant association was present in the total sample population for fruit, total meat, processed meat, coffee, SSB and moderate and heavy alcohol. Fruit intake was not significantly associated with prediabetes, but did show a statistically significant inverse association with UDM and prevalent diabetes, with OR of 0.71 (95\% CI $0.51,0.98)$ and $0.77(95 \%$ CI $0.60,0.98)$ per 1 SD in comparison with NGT, respectively. This association was not present when stratified by sex. Total meat intake was not significantly associated with prediabetes, but showed a statistically significant association with UDM (OR 1.99; 95 $\%$ CI 1.31, 3.00) and prevalent diabetes (OR 1.59; $95 \%$ CI $1 \cdot 13,2 \cdot 24)$ per 1 SD in comparison with NGT. When stratified by sex, a significant association remained only for UDM in men (OR 1.97; $95 \%$ CI 1.23, 3.14) and for prevalent diabetes in women (OR 1.54; $95 \%$ CI 1.03, 2.29) in comparison with NGT. A significant association with UDM and prevalent diabetes was also present for processed meat, with OR per $1 \mathrm{SD}$ of $1.92(95 \%$ CI 1.38, 2.67) and 1.61 (95\% CI 1.20, 2.15), respectively. After stratification for sex, processed meat was significantly associated with UDM in men (OR 2.03; $95 \%$ CI $1.34,3.07)$ and with prevalent diabetes in both men and women (OR 1.53, $95 \%$ CI 1.06, 2.19; and OR 1.58, $95 \%$ CI $1 \cdot 12,2 \cdot 23$, respectively). In addition, SSB intake was significantly positively associated with prevalent diabetes in the total population (OR 1.17; $95 \%$ CI 1.04, 1.33) and in men (OR 1.35 ; $95 \%$ CI 1.00, 1.82). Moderate alcohol consumption was positively associated with UDM in men (OR 2.91; $95 \%$ CI $1 \cdot 10,7 \cdot 71)$.

Concerning diet and prediabetes, coffee consumption was significantly inversely associated with prediabetes (OR 0.86; 


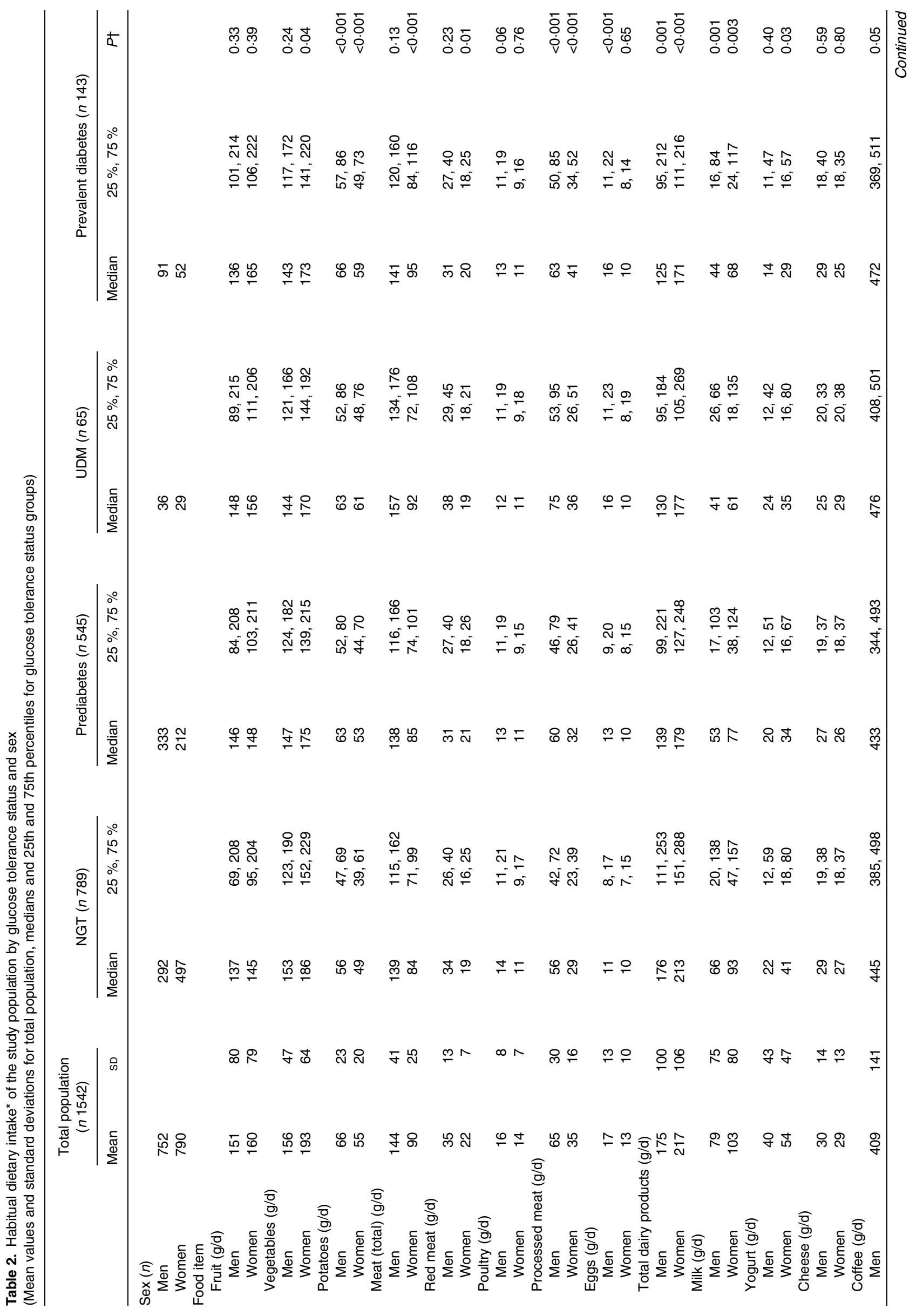




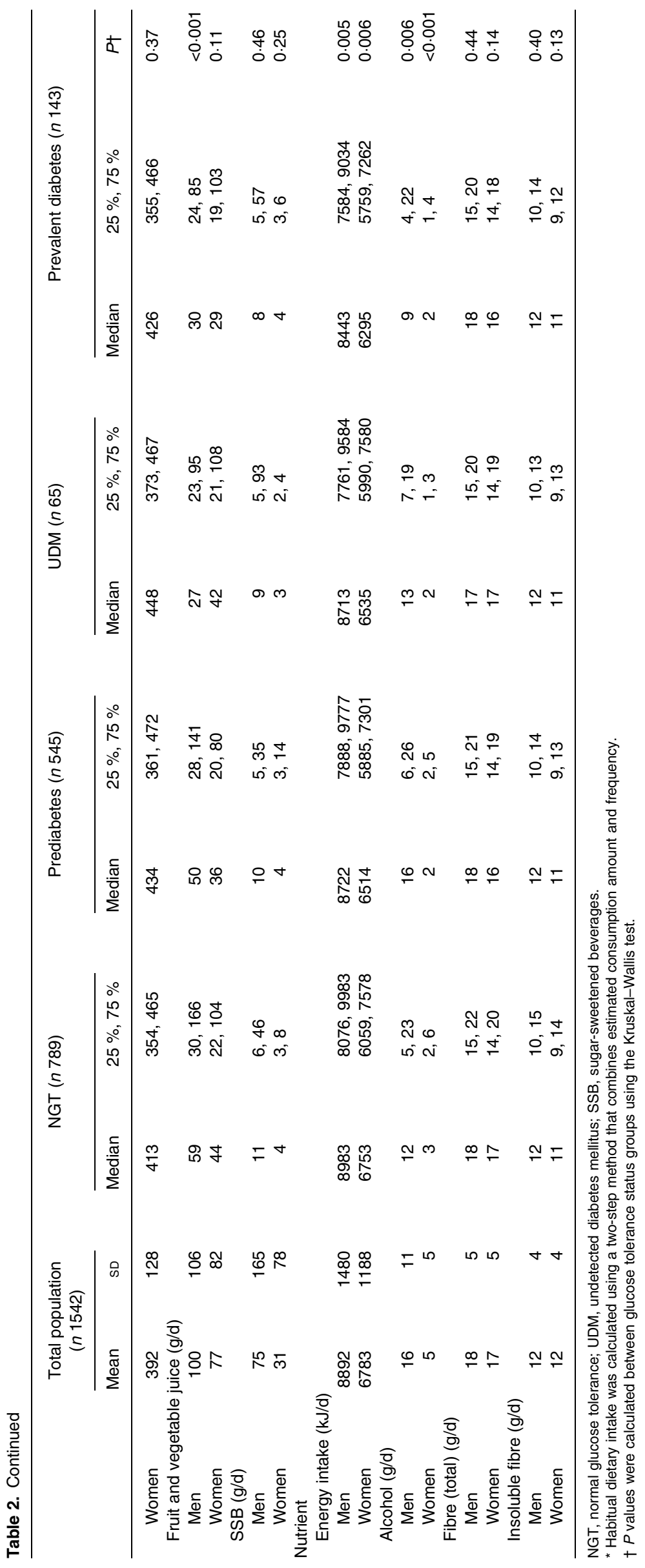


Table 3. Energy-, age- and sex-adjusted associations between the consumption of various foods and nutrients and glucose tolerance status $\dagger$ (Odds ratios and $95 \%$ confidence intervals per 1 standard deviation)

\begin{tabular}{|c|c|c|c|c|c|c|c|}
\hline & \multirow[b]{2}{*}{ SD } & \multicolumn{2}{|c|}{ Prediabetes ( $n$ 545) } & \multicolumn{2}{|c|}{ UDM ( $n$ 65) } & \multicolumn{2}{|c|}{ Prevalent diabetes ( $n$ 143) } \\
\hline & & OR & $95 \% \mathrm{Cl}$ & OR & $95 \% \mathrm{Cl}$ & OR & $95 \% \mathrm{Cl}$ \\
\hline \multicolumn{8}{|l|}{$\operatorname{Sex}(n)$} \\
\hline Men & & \multicolumn{2}{|c|}{333} & \multicolumn{2}{|c|}{36} & \multicolumn{2}{|c|}{91} \\
\hline Women & & \multicolumn{2}{|c|}{212} & \multicolumn{2}{|c|}{29} & \multicolumn{2}{|c|}{52} \\
\hline \multicolumn{8}{|l|}{ Food item } \\
\hline Fruit & 80 & 0.91 & $0.80,1.04$ & $0.72^{*}$ & $0.52,0.98$ & 0.81 & $0.64,1.01$ \\
\hline Men & 80 & 0.89 & $0.75,1.07$ & 0.75 & $0.50,1.15$ & 0.74 & $0.55,1.01$ \\
\hline Women & 79 & 0.95 & $0.78,1.16$ & 0.62 & $0.37,1.03$ & 0.89 & $0.61,1.28$ \\
\hline Vegetables & 59 & 0.92 & $0.80,1.05$ & $0.65^{*}$ & $0.44,0.95$ & 0.98 & $0.77,1.26$ \\
\hline Men & 47 & 0.89 & $0.75,1.06$ & 0.70 & $0.45,1.10$ & 0.80 & $0.59,1.09$ \\
\hline Women & 64 & 0.97 & $0.79,1.18$ & 0.58 & $0.32,1.03$ & 1.20 & $0.85,1.70$ \\
\hline Potatoes & 22 & 1.05 & $0.60,1.82$ & 0.70 & $0.23,2.17$ & 1.21 & $0.54,2.72$ \\
\hline Men & 23 & 1.43 & $0.67,3.05$ & 0.70 & $1.51,3.23$ & 1.53 & $0.54,4.33$ \\
\hline Women & 20 & 0.71 & $0.31,1.65$ & 0.76 & $0.14,4 \cdot 10$ & 0.92 & $0.24,3.51$ \\
\hline Meat (total) & 43 & $1.57^{\star}$ & $1.32,1.88$ & $3 \cdot 14^{*}$ & $2 \cdot 26,4 \cdot 37$ & $2.89^{*}$ & $2 \cdot 20,3 \cdot 80$ \\
\hline Men & 41 & $1 \cdot 35^{\star}$ & $1.10,1.65$ & $2 \cdot 67^{*}$ & $1.86,3.85$ & $2 \cdot 21^{*}$ & $1.64,2.98$ \\
\hline Women & 25 & $1.46^{*}$ & $1.21,1.76$ & $2 \cdot 17^{*}$ & $1.49,3.16$ & $2.65^{*}$ & $1.96,3.58$ \\
\hline Red meat & 12 & $1 \cdot 15$ & $0.996,1.33$ & $1.43^{*}$ & $1.06,1.92$ & $1 \cdot 28^{*}$ & $1.01,1.63$ \\
\hline Men & 13 & 1.03 & $0.87,1.22$ & $1.47^{*}$ & $1.05,2.04$ & 1.20 & $0.92,1.58$ \\
\hline Women & 7 & $1 \cdot 30^{*}$ & $1 \cdot 10,1.54$ & 1.09 & $0.71,1.67$ & 1.29 & $0.94,1.76$ \\
\hline Poultry & 8 & 0.99 & $0.88,1.12$ & $1 \cdot 11$ & $0.85,1.47$ & 1.14 & $0.93,1.40$ \\
\hline Men & 8 & 0.98 & $0.83,1.16$ & 1.06 & $0.72,1.55$ & $1 \cdot 15$ & $0.89,1.50$ \\
\hline Women & 7 & 1.00 & $0.84,1.21$ & 1.19 & $0.80,1.75$ & $1 \cdot 12$ & $0.81,1.55$ \\
\hline Processed meat & 28 & $1.54^{\star}$ & $1.30,1.82$ & $2 \cdot 80^{*}$ & $2.12,3.70$ & $2 \cdot 60^{*}$ & $2.05,3.31$ \\
\hline Men & 30 & $1.44^{*}$ & $1.17,1.77$ & $2 \cdot 68^{*}$ & $1.91,3.78$ & $2 \cdot 25^{*}$ & $1.68,3.02$ \\
\hline Women & 16 & $1.37^{\star}$ & $1.13,1.66$ & $2.09^{*}$ & $1.50,2.91$ & $2.43^{*}$ & $1.84,3.20$ \\
\hline Eggs & 12 & 1.08 & $0.95,1.22$ & $1 \cdot 11$ & $0.88,1.41$ & 1.06 & $0.87,1.28$ \\
\hline Men & 13 & 1.13 & $0.94,1.36$ & $1 \cdot 10$ & $0.78,1.56$ & $1 \cdot 10$ & $0.85,1.44$ \\
\hline Women & 10 & 1.03 & $0.87,1.22$ & 1.15 & $0.84,1.57$ & 1.00 & $0.74,1.35$ \\
\hline Total dairy products & 105 & $0.86^{*}$ & $0.75,0.98$ & 0.75 & $0.54,1.04$ & 0.90 & $0.72,1.14$ \\
\hline Men & 100 & 0.88 & $0.74,1.05$ & 0.67 & $0.42,1.07$ & 0.94 & $0.71,1.25$ \\
\hline Women & 106 & 0.84 & $0.68,1.03$ & 0.84 & $0.52,1.35$ & 0.83 & $0.57,1.23$ \\
\hline Milk & 78 & 0.85 & $0.72,1.00$ & 0.73 & $0.48,1.10$ & 0.83 & $0.62,1.11$ \\
\hline Men & 75 & 0.83 & $0.66,1.03$ & 0.62 & $0.35,1.12$ & 0.73 & $0.50,1.06$ \\
\hline Women & 80 & 0.88 & $0.69,1.12$ & 0.84 & $0.48,1.49$ & 0.97 & $0.64,1.48$ \\
\hline Yogurt & 45 & $0.87^{\star}$ & $0.77,0.99$ & 0.95 & $0.71,1.26$ & 0.97 & $0.79,1.21$ \\
\hline Men & 43 & 0.94 & $0.80,1.12$ & 1.03 & $0.72,1.48$ & $1 \cdot 17$ & $0.92,1.49$ \\
\hline Women & 47 & $0.81^{*}$ & $0.66,0.99$ & 0.86 & $0.54,1.36$ & 0.67 & $0.44,1.03$ \\
\hline Cheese & 13 & 0.95 & $0.59,1.53$ & 0.84 & $0.29,2.45$ & $1 \cdot 71$ & $0.79,3.67$ \\
\hline Men & 14 & 0.74 & $0.40,1.39$ & 0.26 & $0.55,1.21$ & 1.47 & $0.56,3.82$ \\
\hline Women & 13 & 1.34 & $0.65,2.79$ & 3.39 & $0.72,16.00$ & 1.65 & $0.44,6.24$ \\
\hline Coffee & 134 & $0.88^{\star}$ & $0.78,0.99$ & 0.98 & $0.75,1.29$ & 0.87 & $0.72,1.05$ \\
\hline Men & 141 & $0.80^{*}$ & $0.67,0.95$ & 1.03 & $0.71,1.49$ & 0.90 & $0.70,1.17$ \\
\hline Women & 128 & 0.98 & $0.82,1.17$ & 0.90 & $0.61,1.33$ & 0.77 & $0.58,1.03$ \\
\hline SSB & 95 & 1.04 & $0.97,1.13$ & $1 \cdot 19^{\star}$ & $1.01,1.39$ & $1 \cdot 24^{*}$ & $1.12,1.39$ \\
\hline Men & 106 & 1.05 & $0.88,1.24$ & 1.41 & $0.98,2.02$ & $1.54^{*}$ & $1.19,1.99$ \\
\hline Women & 82 & 1.10 & $0.93,1.31$ & $1 \cdot 17$ & $0.77,1.80$ & $1.37^{\star}$ & $1.06,1.76$ \\
\hline Fruit and vegetable juice & 130 & 1.01 & $0.89,1.15$ & 1.01 & $0.75,1.36$ & 1.02 & $0.82,1.28$ \\
\hline Men & 165 & 0.98 & $0.83,1.16$ & 0.86 & $0.55,1.33$ & 0.87 & $0.63,1.19$ \\
\hline Women & 78 & 1.03 & $0.86,1.25$ & 1.23 & $0.83,1.82$ & 1.30 & $0.94,1.79$ \\
\hline \multicolumn{8}{|l|}{ Nutrient } \\
\hline Moderate alcoholf & 10 & 0.79 & $0.60,1.05$ & 0.73 & $0.40,1.32$ & $0.57^{*}$ & $0.36,0.89$ \\
\hline Men & 11 & 1.05 & $0.68,1.62$ & 1.95 & $0.78,4.91$ & 0.91 & $0.48,1.71$ \\
\hline Women & 5 & 0.70 & $0.49,1.01$ & $0.31^{*}$ & $0.13,0.75$ & $0.40^{*}$ & $0.21,0.77$ \\
\hline Heavy alcoholf & 10 & $1 \cdot 22$ & $0.87,1.72$ & 0.67 & $0.30,1.52$ & 0.72 & $0.41,1.27$ \\
\hline Men & 11 & $1.65^{\star}$ & $1.05,2.59$ & $1 \cdot 14$ & $0.38,3.38$ & $1 \cdot 11$ & $0.56,2.20$ \\
\hline Women & 5 & 0.86 & $0.47,1.57$ & 0.86 & $0.23,3.26$ & 0.34 & $0.08,1.55$ \\
\hline Fibre (total) & 5 & $0.74^{\star}$ & $0.63,0.88$ & $0.42^{*}$ & $0.28,0.66$ & $0.64^{*}$ & $0.47,0.87$ \\
\hline Men & 5 & $0.75^{\star}$ & $0.60,0.93$ & $0.41^{*}$ & $0.23,0.74$ & $0.58^{\star}$ & $0.39,0.87$ \\
\hline Women & 5 & $0.76^{*}$ & $0.58,0.99$ & $0.39^{*}$ & $0.20,0.78$ & 0.72 & $0.43,1.21$ \\
\hline Insoluble fibre & 4 & $0.74^{*}$ & $0.63,0.87$ & $0.42^{*}$ & $0.27,0.65$ & $0.65^{*}$ & $0.48,0.88$ \\
\hline Men & 4 & $0.74^{\star}$ & $0.59,0.92$ & $0.41^{*}$ & $0.23,0.73$ & $0.61^{*}$ & $0.42,0.90$ \\
\hline Women & 4 & $0.77^{*}$ & $0.59,0.99$ & $0.39^{*}$ & $0.20,0.77$ & 0.69 & $0.41,1.15$ \\
\hline
\end{tabular}

UDM, undetected diabetes mellitus; SSB, sugar-sweetened beverages.

* Significant result $(P<0.05)$.

† Models were adjusted for age and energy intake; additionally, models were either adjusted for sex or stratified by sex. Normal glucose tolerance status is the reference group. Habitual dietary intake was calculated using a two-step method that combines estimated consumption amount and frequency.

$\ddagger$ Moderate considered $\geq 5 \mathrm{~g} / \mathrm{d}-<20 \mathrm{~g} / \mathrm{d}$ for men, $\geq 2 \mathrm{~g} / \mathrm{d}-<10 \mathrm{~g} / \mathrm{d}$ for women; heavy considered $\geq 20 \mathrm{~g} / \mathrm{d}$ for men, $\geq 10 \mathrm{~g} / \mathrm{d}$ for women. 
Table 4. Fully adjusted associations between the consumption of various foods and nutrients and glucose tolerance statust (Odds ratios and $95 \%$ confidence intervals per 1 standard deviation)

\begin{tabular}{|c|c|c|c|c|c|c|c|}
\hline & \multirow[b]{2}{*}{$\mathrm{SD}$} & \multicolumn{2}{|c|}{ Prediabetes ( $n$ 545) } & \multicolumn{2}{|c|}{ UDM ( $n$ 65) } & \multicolumn{2}{|c|}{ Prevalent diabetes ( $n$ 143) } \\
\hline & & OR & $95 \% \mathrm{Cl}$ & OR & $95 \% \mathrm{Cl}$ & OR & $95 \% \mathrm{Cl}$ \\
\hline \multicolumn{8}{|l|}{$\operatorname{Sex}(n)$} \\
\hline Men & & \multicolumn{2}{|c|}{333} & \multicolumn{2}{|c|}{36} & \multicolumn{2}{|c|}{91} \\
\hline Women & & \multicolumn{2}{|c|}{212} & \multicolumn{2}{|c|}{29} & \multicolumn{2}{|c|}{52} \\
\hline \multicolumn{8}{|l|}{ Food item } \\
\hline Fruit & 80 & 0.90 & $0.78,1.03$ & $0.71^{*}$ & $0.51,0.98$ & $0.77^{\star}$ & $0.60,0.98$ \\
\hline Men & 80 & 0.89 & $0.73,1.08$ & 0.72 & $0.47,1.12$ & 0.74 & $0.53,1.02$ \\
\hline Women & 79 & 0.91 & $0.74,1.12$ & 0.61 & $0.36,1.03$ & 0.78 & $0.51,1.18$ \\
\hline Vegetables & 59 & 1.01 & $0.86,1.18$ & 0.84 & $0.57,1.25$ & $1 \cdot 30$ & $0.99,1.70$ \\
\hline Men & 47 & 0.98 & $0.81,1.20$ & 1.02 & $0.65,1.59$ & $1 \cdot 17$ & $0.83,1.63$ \\
\hline Women & 64 & 1.07 & $0.85,1.34$ & 0.62 & $0.32,1.17$ & 1.47 & $0.98,2.19$ \\
\hline Potatoes & 22 & $1 \cdot 16$ & $0.64,2.09$ & 0.76 & $0.23,2.51$ & 1.73 & $0.72,4.21$ \\
\hline Men & 23 & 1.79 & $0.80,4.00$ & 1.08 & $0.22,5.46$ & 2.93 & $0.93,9.27$ \\
\hline Women & 20 & 0.69 & $0.28,1.70$ & 0.77 & $0.13,4.59$ & 1.01 & $0.22,4.67$ \\
\hline Meat (total) & 43 & 1.01 & $0.81,1.26$ & $1.99^{*}$ & $1.31,3.00$ & $1.59^{*}$ & $1 \cdot 13,2 \cdot 24$ \\
\hline Men & 41 & 1.01 & $0.80,1.28$ & $1.97^{\star}$ & $1 \cdot 23,3 \cdot 14$ & 1.40 & $0.96,2.06$ \\
\hline Women & 25 & 0.96 & $0.76,1.23$ & 1.32 & $0.82,2.10$ & $1.54^{*}$ & $1.03,2.29$ \\
\hline Red meat & 12 & 0.96 & $0.82,1.12$ & $1 \cdot 10$ & $0.79,1.54$ & 0.96 & $0.73,1.27$ \\
\hline Men & 13 & 0.90 & $0.75,1.09$ & $1 \cdot 14$ & $0.78,1.65$ & 0.92 & $0.67,1.26$ \\
\hline Women & 7 & $1 \cdot 10$ & $0.91,1.33$ & 0.86 & $0.53,1.40$ & 0.96 & $0.66,1.40$ \\
\hline Poultry & 8 & 0.94 & $0.83,1.07$ & 1.06 & $0.79,1.43$ & $1 \cdot 11$ & $0.88,1.38$ \\
\hline Men & 8 & 0.96 & $0.81,1.14$ & 1.07 & $0.71,1.60$ & $1 \cdot 16$ & $0.87,1.55$ \\
\hline Women & 7 & 0.91 & $0.75,1.11$ & 1.05 & $0.69,1.62$ & 0.96 & $0.65,1.42$ \\
\hline Processed meat & 28 & 1.09 & $0.90,1.32$ & $1.92^{*}$ & $1 \cdot 38,2 \cdot 67$ & $1 \cdot 61^{*}$ & $1 \cdot 20,2 \cdot 15$ \\
\hline Men & 30 & $1 \cdot 15$ & $0.91,1.45$ & $2.03^{*}$ & $1.34,3.07$ & $1.53^{*}$ & $1.06,2.19$ \\
\hline Women & 16 & 0.92 & $0.72,1.17$ & 1.33 & $0.89,1.98$ & $1.58^{*}$ & $1 \cdot 12,2 \cdot 23$ \\
\hline Eggs & 12 & 1.04 & $0.91,1.18$ & 1.05 & $0.81,1.34$ & 0.95 & $0.77,1.18$ \\
\hline Men & 13 & 1.07 & $0.88,1.29$ & 1.06 & $0.73,1.55$ & 1.00 & $0.75,1.34$ \\
\hline Women & 10 & 0.99 & $0.82,1.19$ & 1.05 & $0.75,1.47$ & 0.88 & $0.63,1.24$ \\
\hline Total dairy products & 105 & 0.88 & $0.77,1.02$ & 0.78 & $0.56,1.09$ & 0.94 & $0.73,1.20$ \\
\hline Men & 100 & 0.92 & $0.77,1.11$ & 0.75 & $0.47,1.19$ & 1.02 & $0.75,1.37$ \\
\hline Women & 106 & 0.87 & $0.70,1.08$ & 0.83 & $0.51,1.34$ & 0.77 & $0.50,1.17$ \\
\hline Milk & 78 & 0.87 & $0.73,1.04$ & 0.73 & $0.47,1.11$ & 0.85 & $0.62,1.16$ \\
\hline Men & 75 & 0.89 & $0.71,1.12$ & 0.71 & $0.39,1.29$ & 0.79 & $0.53,1.19$ \\
\hline Women & 80 & 0.86 & $0.66,1.12$ & 0.76 & $0.42,1.37$ & 0.84 & $0.52,1.34$ \\
\hline Yogurt & 45 & 0.88 & $0.77,1.01$ & 0.94 & $0.71,1.26$ & 0.96 & $0.77,1.21$ \\
\hline Men & 43 & 0.92 & $0.77,1.10$ & 0.97 & $0.67,1.39$ & $1 \cdot 12$ & $0.87,1.45$ \\
\hline Women & 47 & 0.85 & $0.69,1.05$ & 0.91 & $0.56,1.49$ & 0.64 & $0.40,1.04$ \\
\hline Cheese & 13 & 0.97 & $0.58,1.61$ & 1.02 & $0.33,3.15$ & 2.02 & $0.87,4.69$ \\
\hline Men & 14 & 0.68 & $0.35,1.33$ & 0.29 & $0.06,1.49$ & 1.92 & $0.64,5.74$ \\
\hline Women & 13 & 1.65 & $0.74,3.67$ & $4 \cdot 14$ & $0.83,20.76$ & 2.07 & $0.47,9.04$ \\
\hline Coffee & 134 & $0.86^{\star}$ & $0.76,0.98$ & 0.98 & $0.74,1.30$ & 0.85 & $0.69,1.05$ \\
\hline Men & 141 & $0.80^{*}$ & $0.67,0.96$ & 1.01 & $0.68,1.49$ & 0.84 & $0.63,1.11$ \\
\hline Women & 128 & 0.94 & $0.77,1.14$ & 0.90 & $0.60,1.36$ & 0.80 & $0.57,1.12$ \\
\hline SSB & 95 & 1.00 & $0.92,1.08$ & $1 \cdot 11$ & $0.93,1.32$ & $1 \cdot 17^{*}$ & $1.04,1.33$ \\
\hline Men & 106 & 0.96 & $0.79,1.16$ & $1 \cdot 17$ & $0.78,1.76$ & $1 \cdot 35^{\star}$ & $1.00,1.82$ \\
\hline Women & 82 & 1.05 & $0.86,1.28$ & $1 \cdot 10$ & $0.67,1.80$ & 1.33 & $0.97,1.82$ \\
\hline Fruit and vegetable juice & 130 & 0.97 & $0.85,1.10$ & 0.94 & $0.69,1.26$ & 0.92 & $0.72,1.17$ \\
\hline Men & 165 & 0.97 & $0.81,1.16$ & 0.82 & $0.52,1.27$ & 0.82 & $0.59,1.15$ \\
\hline Women & 78 & 0.96 & $0.78,1.16$ & 1.08 & $0.72,1.63$ & 1.08 & $0.75,1.54$ \\
\hline \multicolumn{8}{|l|}{ Nutrient } \\
\hline Moderate alcoholł & 10 & $1 \cdot 16$ & $0.85,1.57$ & $1 \cdot 36$ & $0.72,2.57$ & 1.05 & $0.64,1.71$ \\
\hline Men & 11 & 1.22 & $0.77,1.93$ & $2.91^{*}$ & $1 \cdot 10,7 \cdot 71$ & $1 \cdot 30$ & $0.65,2.60$ \\
\hline Women & 5 & $1 \cdot 22$ & $0.80,1.85$ & 0.59 & $0.22,1.55$ & 0.96 & $0.45,2.03$ \\
\hline Heavy alcoholł & 10 & $1 \cdot 78^{*}$ & $1.23,2.59$ & $1 \cdot 22$ & $0.52,2.86$ & $1 \cdot 26$ & $0.67,2.36$ \\
\hline Men & 11 & $1.84^{*}$ & $1.14,2.95$ & 1.52 & $0.49,4.72$ & 1.45 & $0.69,3.04$ \\
\hline Women & 5 & 1.81 & $0.91,3.62$ & $1 \cdot 71$ & $0.41,7.16$ & 0.77 & $0.15,4.06$ \\
\hline Fibre (total) & 5 & 0.91 & $0.75,1.11$ & 0.64 & $0.40,1.04$ & 1.02 & $0.72,1.44$ \\
\hline Men & 5 & 0.91 & $0.70,1.17$ & 0.66 & $0.35,1.24$ & 0.96 & $0.61,1.50$ \\
\hline Women & 5 & 0.95 & $0.69,1.29$ & 0.55 & $0.25,1.22$ & $1 \cdot 22$ & $0.67,2.23$ \\
\hline Insoluble fibre & 4 & 0.90 & $0.75,1.09$ & 0.63 & $0.40,1.01$ & 1.03 & $0.74,1.45$ \\
\hline Men & 4 & 0.89 & $0.69,1.14$ & 0.64 & $0.34,1.20$ & 0.98 & $0.63,1.52$ \\
\hline Women & 4 & 0.95 & $0.71,1.29$ & 0.54 & $0.24,1.18$ & $1 \cdot 21$ & $0.67,2.19$ \\
\hline
\end{tabular}

UDM, undetected diabetes mellitus; SSB, sugar-sweetened beverages.

* Significant result $(P<0.05)$.

† Models were adjusted for age, energy intake, BMI, waist circumference, family history of diabetes, physical activity, smoking, education level and hypertension; additionally, models were either adjusted for sex or stratified by sex. Normal glucose tolerance status is the reference group. Habitual dietary intake was calculated using a two-step method that combines estimated consumption amount and frequency.

$\ddagger$ Moderate considered $\geq 5 \mathrm{~g} / \mathrm{d}-<20 \mathrm{~g} / \mathrm{d}$ for men, $\geq 2 \mathrm{~g} / \mathrm{d}-<10 \mathrm{~g} / \mathrm{d}$ for women; heavy considered $\geq 20 \mathrm{~g} / \mathrm{d}$ for men, $\geq 10 \mathrm{~g} / \mathrm{d}$ for women. 
$95 \%$ CI $0 \cdot 76,0.98)$ per $1 \mathrm{sD}$ in comparison with NGT. A significant association remained only in men once the analysis was stratified by sex (OR 0.80; $95 \%$ CI $0.67,0.96)$. Heavy alcohol intake was positively associated with prediabetes (OR 1.78 ; $95 \%$ CI $1.23,2.59)$, but this association remained statistically significant only for men after stratification by sex (OR 1.84; $95 \%$ CI 1.14, 2.95). While total fibre, insoluble fibre, total dairy product and yogurt intake were inversely associated with prediabetes in the crude models, these associations were no longer statistically significant in the fully adjusted models (though risk estimates remained similar).

\section{Discussion}

As a high percentage of individuals with prediabetes may progress to developing T2DM, a widespread disease with high personal and economic costs, it is imperative that interventions be focused not only on managing T2DM, but also on primary prevention $^{(1-3)}$. Several studies have already demonstrated the effectiveness of diet and lifestyle interventions in slowing or preventing the progression of prediabetes to diabetes ${ }^{(6,7)}$. Additionally, the state of prediabetes may already be associated with negative outcomes ${ }^{(5)}$. Therefore, a full understanding of the interaction between diet and the development and progression of both prediabetes and diabetes is vital. A few studies have analysed the relationship between certain foods and the risk of prediabetes, but to our knowledge, the present study is the first to examine the associations between multiple food groups and prediabetes in a large sample ${ }^{(8-12)}$.

In the present study, fruit was inversely associated with UDM and prevalent diabetes, while total meat, processed meat, SSB and moderate alcohol were positively associated with UDM and/or prevalent diabetes. Coffee intake was inversely associated with prediabetes while heavy alcohol consumption was positively associated with prediabetes. These results are largely consistent with what is found in the existing literature $e^{(11,12,20,23,35)}$. In contrast to previous findings, red meat, vegetables, fibre (total and insoluble), fruit and vegetable juice, total dairy products, milk, yogurt, cheese, poultry, eggs and potatoes were not associated with glucose tolerance status in this population after adjustment for all covariates $^{(20,23,25,27-29,31,35-38)}$. Overall, significant associations were seen more frequently in the total sample than when split by sex, probably because of the larger sample size, and more often in men than women.

Coffee intake was significantly inversely associated with prediabetes in the study population as a whole and in men when stratified by sex. A moderate, non-significant trend towards decreased risk was seen in most glucose tolerance categories. These results are consistent with a previous study by Lee et al. ${ }^{(11)}$, which found an inverse association between coffee intake and the risk of incident prediabetes and T2DM in individuals with known T2DM-related genetic mutations. Further studies have reported that coffee may lower the risk of developing T2DM or reduce the likelihood of progression from prediabetes to diabetes ${ }^{(23,26,39,40)}$. However, no previous study to our knowledge has identified an association between coffee consumption and prediabetes in a population-based sample.
Heavy alcohol intake was significantly positively associated with prediabetes in the total study population and in men, while a non-significant trend towards increased risk was present in all other groups except for women with prevalent diabetes. This is consistent with the results of one cohort study which found an increased risk of incident prediabetes and T2DM in men with heavy total alcohol intake, as well as two meta-analyses which found that heavy alcohol intake was associated with an increased risk of diabetes ${ }^{(12,22,41)}$. However, some studies have found that high alcohol intake had no effect on T2DM incidence ${ }^{(42-44)}$. In sensitivity analyses using $14 \mathrm{~g} / \mathrm{d}$ (approximately $100 \mathrm{~g}$ per week) as the cut-off between moderate and heavy intake for both men and women, results were not significantly different ${ }^{(45)}$.

Several meta-analyses suggest that moderate alcohol intake, on the other hand, may be protective against prediabetes or $\mathrm{T}_{2} \mathrm{DM}^{(41-44,46)}$. However, moderate alcohol consumption was positively associated with UDM in men in our study. This is in accordance with a recent meta-analysis that found a modest increase in diabetes risk for men starting at a relatively low alcohol consumption level. In the same study, a protective effect of moderate alcohol intake was seen in women $^{(22)}$. Cullman et al. ${ }^{(12)}$ also found that moderate alcohol consumption was protective in women, but not men. In our study, moderate alcohol was significantly inversely associated with prevalent diabetes and UDM in women in the crude analyses. Significance did not persist after full adjustment, but a non-significant inverse association did remain between moderate alcohol and UDM and prevalent diabetes in women. A potential reason why these results did not reach significance is that it was not possible to distinguish 'former drinkers' and 'never drinkers' from the reference category. As former drinkers are more likely to exhibit other health problems, therefore resulting in abstinence from alcohol, they would ideally be separated from the reference group ${ }^{(47)}$. Additionally, it should be noted that many studies have used cut-offs of between 30 and $60 \mathrm{~g} / \mathrm{d}$ as the line between moderate and heavy alcohol intake. These levels are considerably higher than the cut-offs used in this study, which were chosen to align with actual alcohol consumption recommendations ${ }^{(22,41,43,44,46)}$.

In the crude analyses, total dairy products (total population) and yogurt (total population and in women) were significantly inversely associated with prediabetes. Although significance did not persist after full adjustment, a trend towards decreased risk of prediabetes remained for total dairy products, milk and yogurt in almost all categories. This is in line with a recent study that found that total dairy products, milk and yogurt were significantly associated with a decreased risk of incident prediabetes ${ }^{(8)}$. A number of meta-analyses have also found a significant inverse association between dairy products and the risk of diabetes ${ }^{(33,37,48)}$. However, results have been inconsistent in the literature, and we were not able to confirm an association between dairy product intake and glucose tolerance status after final adjustment for covariates.

The German Diabetes Risk Score, developed by the German Institute of Human Nutrition Potsdam-Rehbruecke (DIfE), considers age, height, high blood pressure, a family history of 
diabetes, waist circumference, physical activity, whole grain intake, meat intake, coffee consumption and smoking as major factors contributing to diabetes risk ${ }^{(49)}$. All of these risk factors were also taken into account in the present analyses. Older age, higher BMI, high blood pressure, positive family history of diabetes, larger waist circumference and inactivity all were more prevalent in individuals with prediabetes, UDM or prevalent diabetes than NGT. Smoking, however, was most prevalent in participants with NGT. Total meat and processed meat were significantly positively associated with UDM and prevalent diabetes, but red meat was not significantly associated with glucose tolerance status in this sample. A potential explanation for this discrepancy is the relatively low consumption of red meat in this population (Table 2$)^{(27,35)}$. Insoluble fibre was taken as a potential proxy variable for whole grain intake, but while total fibre and insoluble fibre were significantly associated with reduced risk in nearly all glucose tolerance status categories in the crude analyses, neither insoluble nor total fibre was significantly associated with glucose tolerance status after adjusting for major potential covariates. These results are in accordance with a 2015 meta-analysis that found that intakes of total, cereal and vegetable fibre were significantly associated with a decreased risk of T2DM, but that the associations were no longer significant after adjustment for $\mathrm{BMI}^{(24)}$. Several other meta-analyses found that whole grain and/or dietary fibre intake to be associated with a lower risk of $\mathrm{T} 2 \mathrm{DM}^{(38,50,51)}$.

Because of the cross-sectional nature of this study, these results cannot be interpreted as determining a causal relationship. Strengths of this study include a large sample size, which was initially randomly selected from the general population, and the large number of food items evaluated in this study. Other strengths include the use of an oral glucose tolerance test or physician-confirmed diagnosis to determine glucose tolerance status, the large number of covariates adjusted for, the consideration of sex-specific differences and the use of a sophisticated National Cancer Institute-based method for dietary intake evaluation ${ }^{(17,18)}$. In this study, participants with prediabetes, UDM or prevalent T2DM reported lower energy intake than participants with NGT, despite having a higher BMI (Table 2). This is an indicator that under-reporting of energy intake may be present, which could affect results. However, this may be partially explained by the higher physical activity of participants with NGT and the possibility that participants with prevalent T2DM were advised by their physicians to reduce their energy consumption in attempt to achieve a healthy weight. Such a change in dietary patterns could also have a resulting effect on the cross-sectional dietdiabetes association. However, we believe it is unlikely, as habitual dietary intake and the BMI of participants with prevalent T2DM and UDM (who would not have been advised to alter their diet) are very comparable. Still, this is one reason that confirmation with longitudinal studies is important. Selection bias as a result of the reduced size of the final study sample (due to lower participation in KORA FF4 than S4, as well as exclusion criteria in this analysis) could be another potential limitation. Lastly, our results have not been adjusted for multiple testing. The power would not have been sufficient to detect relevant associations in the fully adjusted analysis because of the relatively small number of participants in each group after stratification by glucose tolerance status and sex. However, when the conservative Bonferroni correction method is applied, those results with the smallest $P$ values still remain statistically significant (total meat, processed meat and heavy alcohol; results not shown).

In conclusion, coffee consumption was significantly inversely associated with prediabetes, while heavy alcohol intake was significantly positively associated with prediabetes. Fruit consumption was inversely associated with UDM and prevalent T2DM. Alternatively, total meat, processed meat, SSB and moderate alcohol consumption were significantly positively associated with UDM and/or prevalent diabetes in this analysis. For the first time to our knowledge, we have examined associations between multiple food groups and prevalent prediabetes in a large sample. We have also identified, for the first time, a significant inverse association between coffee and prediabetes in a population-based sample. Additionally, consistent with a previous meta-analysis, our results suggest that moderate alcohol intake may be positively associated with T2DM in men, and we have replicated previous findings regarding a positive association between heavy alcohol consumption and prediabetes $^{(12,22)}$. As dietary factors are modifiable, they are ideal targets for the primary prevention of T2DM. Our findings regarding coffee and heavy alcohol intake highlight potential targets for the primary prevention of prediabetes, in particular. These results should be taken into account in the planning of further studies, especially those experimental and prospective in nature, in order to further clarify these relationships.

\section{Acknowledgements}

Dietary assessment in KORA FF4 was supported by iMED, a research alliance within the Helmholtz Association, Germany. The preparation of this paper was supported by the enableCluster and is catalogued by the enable Steering Committee as enable 032 (http://enable-cluster.de). This work was funded by a grant of the German Federal Ministry for Education and Research (BMBF) (FK 01EA1409E). The KORA study was initiated and financed by the Helmholtz Zentrum München German Research Center for Environmental Health, which is funded by the BMBF and by the State of Bavaria. The funding agencies had no role in the design, analysis or writing of this article.

J. L., A. R. and T. A. B. formulated the research question and designed the study; B. T., C. M., A. P., W. R., K. S., A. Q. and J. L. conducted research and/or provided essential materials; T. A. B. analysed data, performed statistical analysis and wrote the paper; N. W. contributed to statistical analysis and interpretation. All authors read and approved the final manuscript.

There were no conflicts of interest.

\section{References}

1. Müller N, Heller T, Freitag MH, et al. (2015) Healthcare utilization of people with type 2 diabetes in Germany: an analysis based on health insurance data. Diabet Med 32, 951-957. 
2. Wu Y, Ding Y, Tanaka Y, et al. (2014) Risk factors contributing to type 2 diabetes and recent advances in the treatment and prevention. Int J Med Sci 11, 1185-1200.

3. Fowler MJ (2008) Microvascular and macrovascular complications of diabetes. Clin Diabetes 26, 77-82.

4. Buysschaert M \& Bergman M (2011) Definition of prediabetes. Med Clin North Am 95, 289-297, vii.

5. Brannick B, Wynn A \& Dagogo-Jack S (2016) Prediabetes as a toxic environment for the initiation of microvascular and macrovascular complications. Exp Biol Med (Maywood) 241, 1323-1331.

6. Tabák AG, Herder C, Rathmann W, et al. (2012) Prediabetes: a high-risk state for diabetes development. Lancet 379, 2279-2290.

7. Heidemann C, Du Y, Paprott R, et al. (2016) Temporal changes in the prevalence of diagnosed diabetes, undiagnosed diabetes and prediabetes: findings from the German health interview and examination surveys in 1997-1999 and 2008-2011. Diabet Med 33, 14061414.

8. Hruby A, Ma J, Rogers G, et al. (2017) Associations of dairy intake with incident prediabetes or diabetes in middle-aged adults vary by both dairy type and glycemic status. J Nutr 147, 1764-1775.

9. Wirström T, Hilding A, Gu HF, et al. (2013) Consumption of whole grain reduces risk of deteriorating glucose tolerance, including progression to prediabetes. Am J Clin Nutr 97, 179-187.

10. Ma J, Jacques PF, Meigs JB, et al. (2016) Sugar-sweetened beverage but not diet soda consumption is positively associated with progression of insulin resistance and prediabetes. J Nutr 146, 2544-2550.

11. Lee JK, Kim K, Ahn Y, et al. (2015) Habitual coffee intake, genetic polymorphisms, and type 2 diabetes. Eur J Endocrinol 172, 595-601.

12. Cullmann M, Hilding A \& Östenson CG (2012) Alcohol consumption and risk of pre-diabetes and type 2 diabetes development in a Swedish population. Diabet Med 29, 441-452.

13. Kowall B, Rathmann W, Stang A, et al. (2017) Perceived risk of diabetes seriously underestimates actual diabetes risk: the KORA FF4 study. PLOS ONE 12, e0171152.

14. American Diabetes Association (2011) Diagnosis and classification of diabetes mellitus. Diabetes Care 34, S62-S69.

15. Freese J, Feller S, Harttig U, et al. (2014) Development and evaluation of a short 24-h food list as part of a blended dietary assessment strategy in large-scale cohort studies. Eur J Clin Nutr 68, 324-329.

16. Bohlscheid-Thomas S, Hoting I, Boeing H, et al. (1997) Reproducibility and relative validity of energy and macronutrient intake of a food frequency questionnaire developed for the German part of the EPIC project. European Prospective Investigation into Cancer and Nutrition. Int J Epidemiol 26, S71-S81.

17. Kipnis V, Midthune D, Buckman D, et al. (2009) Modeling data with excess zeros and measurement error: application to evaluating relationships between episodically consumed foods and health outcomes. Biometrics 65, 1003-1010.

18. Tooze J, Kipnis V, Buckman D, et al. (2010) A mixed-effects model approach for estimating the distribution of usual intake of nutrients: the NCI method. Stat Med 29, 2857-2868.

19. Slimani N, Deharveng G, Charrondière RU, et al. (1999) Structure of the standardized computerized 24-h diet recall interview used as reference method in the 22 centers participating in the EPIC project. European Prospective Investigation into Cancer and Nutrition. Comput Methods Programs Biomed 58, 251-266.

20. Wang P, Fang J, Gao Z, et al. (2016) Higher intake of fruits, vegetables or their fiber reduces the risk of type 2 diabetes: a meta-analysis. J Diabetes Investig 7, 56-69.

21. Mozaffarian D (2016) Dietary and policy priorities for cardiovascular disease, diabetes, and obesity: a comprehensive review. Circulation 133, 187-225.

22. Knott C, Bell S \& Britton A (2015) Alcohol consumption and the risk of type 2 diabetes: a systematic review and dose-response meta-analysis of more than 1.9 million individuals from 38 observational studies. Diabetes Care 38, 1804-1812.

23. Imamura F, O'Connor L, Ye Z, et al. (2015) Consumption of sugar sweetened beverages, artificially sweetened beverages, and fruit juice and incidence of type 2 diabetes: systematic review, meta-analysis, and estimation of population attributable fraction. Br Med J 351, h3576.

24. InterAct Consortium (2015) Dietary fibre and incidence of type 2 diabetes in eight European countries: the EPIC-InterAct study and a meta-analysis of prospective studies. Diabetologia 58, 1394 1408.

25. Muraki I, Rimm EB, Willett WC, et al. (2016) Potato consumption and risk of type 2 diabetes: results from three prospective cohort studies. Diabetes Care 39, 376-384.

26. Lee JH, Oh MK, Lim JT, et al. (2016) Effect of coffee consumption on the progression of type 2 diabetes mellitus among prediabetic individuals. Korean J Fam Med 37, 7-13.

27. Pan A, Sun Q, Bernstein AM, et al. (2013) Changes in red meat consumption and subsequent risk of type 2 diabetes mellitus: three cohorts of US men and women. JAMA Intern Med 173, 1328-1335.

28. Xi B, Li S, Liu Z, et al. (2014) Intake of fruit juice and incidence of type 2 diabetes: a systematic review and meta-analysis. PLOS ONE 9, e93471.

29. Tamez M, Virtanen JK \& Lajous M (2016) Egg consumption and risk of incident type 2 diabetes: a dose-response meta-analysis of prospective cohort studies. Br J Nutr 115, 2212-2218.

30. Deutsche Gesellschaft für Ernährung (DGE), Österreichische Gesellschaft für Ernährung (ÖGE), Schweizerische Gesellschaft für Ernährungsforschung (SGE), et al. (2008) Referenzwwerte für die Nährstoffzufubr 1. Auflage (Reference Levels for Nutrient Intake, 1st ed.). Neustadt an der Weinstraße: Neuer Umschau Buchverlag.

31. Carter P, Gray LJ, Troughton J, et al. (2010) Fruit and vegetable intake and incidence of type 2 diabetes mellitus: systematic review and meta-analysis. Br Med J 341, c4229.

32. Afshin A, Micha R, Khatibzadeh S, et al. (2014) Consumption of nuts and legumes and risk of incident ischemic heart disease, stroke, and diabetes: a systematic review and meta-analysis. Am J Clin Nutr 100, 278-288.

33. Chen M, Sun Q, Giovannucci E, et al. (2014) Dairy consumption and risk of type 2 diabetes: 3 cohorts of US adults and an updated meta-analysis. BMC Med 12, 215.

34. Rathmann W, Haastert B, Icks A, et al. (2005) Sex differences in the associations of socioeconomic status with undiagnosed diabetes mellitus and impaired glucose tolerance in the elderly population: the KORA Survey 2000. Eur J Public Health 15, 627-633.

35. Micha R, Michas G \& Mozaffarian D (2012) Unprocessed red and processed meats and risk of coronary artery disease and type 2 diabetes an updated review of the evidence. Curr Atheroscler Rep 14, 515-524.

36. Li M, Fan Y, Zhang X, et al. (2014) Fruit and vegetable intake and risk of type 2 diabetes mellitus: meta-analysis of prospective cohort studies. BMJ Open 4, e005497.

37. Gijsbers L, Ding E, Malik V, et al. (2016) Consumption of dairy foods and diabetes incidence: a dose-response meta-analysis of observational studies. Am J Clin Nutr 103, 1111-1124.

38. Yao B, Fang H, Xu W, et al. (2014) Dietary fiber intake and risk of type 2 diabetes: a dose-response analysis of prospective studies. Eur J Epidemiol 29, 79-88.

39. Yarmolinsky J, Mueller NT, Duncan BB, et al. (2015) Coffee consumption, newly diagnosed diabetes, and other alterations in glucose homeostasis: a cross-sectional analysis of the longitudinal study of adult health (ELSA-Brasil). PLOS ONE 10, e0126469.

40. Bhupathiraju SN, Pan A, Manson JE, et al. (2014) Changes in coffee intake and subsequent risk of type 2 diabetes: three large cohorts of US men and women. Diabetologia 57, 1346-1354.

41. Baliunas DO, Taylor BJ, Irving $\mathrm{H}$, et al. (2009) Alcohol as a risk factor for type 2 diabetes: a systematic review and meta-analysis. Diabetes Care 32, 2123-2132.

42. Li XH, Yu FF, Zhou YH, et al. (2016) Association between alcohol consumption and the risk of incident type 2 diabetes: a systematic review and dose-response meta-analysis. Am J Clin Nutr 103, 818-829.

43. Koppes LL, Dekker JM, Hendriks HF, et al. (2005) Moderate alcohol consumption lowers the risk of type 2 diabetes: a meta-analysis of prospective observational studies. Diabetes Care 28, 719-725. 
44. Howard AA, Arnsten JH \& Gourevitch MN (2004) Effect of alcohol consumption on diabetes mellitus: a systematic review. Ann Intern Med 140, 211-219.

45. Wood A, Kaptoge S, Butterworth A, et al. (2018) Risk thresholds for alcohol consumption: combined analysis of individualparticipant data for 599912 current drinkers in 83 prospective studies. Lancet 391, 1513-1523.

46. Carlsson S, Hammar N \& Grill V (2005) Alcohol consumption and type 2 diabetes meta-analysis of epidemiological studies indicates a U-shaped relationship. Diabetologia 48, 1051-1054.

47. Shaper AG, Wannamethee G \& Walker M (1988) Alcohol and mortality in British men: explaining the U-shaped curve. Lancet ii, $1267-1273$.
48. Aune D, Norat T, Romundstad P, et al. (2013) Dairy products and the risk of type 2 diabetes: a systematic review and dose-response meta-analysis of cohort studies. Am J Clin Nutr 98, 1066-1083.

49. Mühlenbruch K, Ludwig T, Jeppesen C, et al. (2014) Update of the German diabetes risk score and external validation in the German MONICA/KORA study. Diabetes Res Clin Pract 104, 459-466.

50. Aune D, Norat T, Romundstad P, et al. (2013) Whole grain and refined grain consumption and the risk of type 2 diabetes: a systematic review and dose-response meta-analysis of cohort studies. Eur J Epidemiol 28, 845-858.

51. Ye E, Chacko S, Chou E, et al. (2012) Greater whole-grain intake is associated with lower risk of type 2 diabetes, cardiovascular disease, and weight gain. J Nutr 142, 1304-1313. 